GANIT J. Bangladesh Math. Soc. (ISSN 1606-3694) 39 (2019) 15-25

DOI: https://doi.org/10.3329/ganit.v39i0.44161

\title{
ON SOME VALUES OF THE SANDOR-SMARANDACHE FUNCTION
}

\author{
A.A.K. Majumdar \\ Ritsumeikan Asia-Pacific University, Beppu-shi 874-8577, Japan \\ E-mail : majumdar@apu.ac.jp
}

Received: 01-08-2018

Accepted: 24-03-2019

\begin{abstract}
Sandor [1] posed a new function, denoted by $S S(n)$, and is defined as follows : $S S(n)=\max \left\{m: 1 \leq m \leq n-1, n \operatorname{divides}\left(\begin{array}{l}n \\ m\end{array}\right)\right\}, n \geq 2\left(\begin{array}{l}n \\ m\end{array}\right)=\frac{n !}{m !(n-m) !}$ being the binomial
\end{abstract} coefficients. This paper finds $S S(n)$ for some particular cases of $\mathrm{n}$.

Keywords : Sandor-Smarandache function, Binomial coefficient, Diophantine equation

\section{Introduction}

Let $C(n, m)$ be the binomial coefficient, defined as follows :

$$
C(n, m)=\left(\begin{array}{c}
n \\
m
\end{array}\right)=\frac{n !}{m !(n-m) !}, O \leq m \leq n .
$$

Then, the Sandor-Smarandache function, denoted by $\operatorname{SS}(n)$, is defined as follows :

$$
S S(n)=\max \left\{m: 1 \leq m \leq n-1, n \text { divides }\left(\begin{array}{c}
n \\
m
\end{array}\right)\right\}, n \geq 3,
$$

with

$$
\operatorname{SS}(1)=1, \operatorname{SS}(2)=1, \operatorname{SS}(3)=1, \operatorname{SS}(4)=1, \operatorname{SS}(6)=1 .
$$

Throughout this paper, we use the following formula for $C(n, m)$ :

$$
C(n, m)=\frac{n(n-1)(n-2) \ldots(n-m+1)}{m !}, O \leq m \leq n .
$$

Sandor [1] proved the result below.

Lemma 1.1: SS $(n)=n-2$ for any odd integer $n(\geq 3)$.

Corollary 1.1: For any prime $p \geq 3, S S(p)=p-2$, and in general,

$$
S S\left(p_{1} p_{2} \ldots p_{s}\right)=p_{1} p_{2} \ldots p_{s}-2 \text { for any odd primes } p_{1}, p_{2}, \ldots, p_{s} .
$$

(C) GANIT: Journal of Bangladesh Mathematical Society, 2019 
As has been pointed out by Sandor [1], to find $S S(n)$, the case of even $n$ is more involving. This paper considers this case in the next section. We first derive $S S(n)$ when $n=p+1, p$ being an odd prime. Next, we confine our attention to the prime $p$ of the four forms, $p=5 v+1, \quad p=5 v+2, p$ $=5 v+3$ and $p=5 v+4$ (for some integer $v \geq 1$ ).

\section{Main Results}

In a recent book, Majumdar [2] derived the expressions of $S S(n)$ for some particular cases. In this section, we derive more to supplement the results found in [2]. We concentrate on the functions $S S(p+1)$, where $p$ is an odd prime of the forms $p=5 v+1,5 v+2,5 v+3,5 v+4$.

We first prove the following simple result.

Lemma 2.1 : Let $p(\geq 5)$ be an odd prime. Then, $S S(p+1)=p-2$

if and only if $p+1$ is not a multiple of 3 .

Proof : We consider

$$
\frac{(p+1) p(p-1)}{3 !}
$$

If 3 does not divide $p+1$, then 3 must divide $p-1$, and hence, 6 divides $p-1$.

Conversely, if $S S(p+1)=p-2$, then 3 must divide $p-1$, and consequently, $p+1$ is not divisible by 3 .

Applying Lemma 2.1, we get the following expressions :

$$
S S(8)=5, \operatorname{SS}(14)=11, S S(20)=17, S S(32)=29, \operatorname{SS}(38)=35, S S(44)=41 .
$$

Corollary 2.1 : Let $p(\geq 5)$ be an odd prime such that 3 divides $p+1$. Then,

$$
S S(p+1) \geq p-3 \text {. }
$$

Proof: follows immediately by virtue of Lemma 2.1 .

Lemma 2.2 - Lemma 2.7 below deal with the case when $p$ is a prime of the form $p=5 v+1$.

Lemma 2.2 : Let $p$ be a prime of the form $p=5 v+1$ for some integer $v \geq 1$. Then,

$$
S S(p+1)=p-3,
$$

if $v=8(3 s+1)$ for some integer $s \geq 0$.

Proof: With $p=5 v+1$, the following expression

$$
(p+1) p \frac{(p-1)(p-2)}{4 !}
$$

takes the form 


$$
5(p+1) p \frac{v(5 v-1)}{2 \times 3 \times 4} .
$$

We consider the case when 8 divides $v$ and 3 divides $5 v-1$, so that

$$
v=8 x, 5 v=3 y+1 \text { for some integers } x \geq 1, y \geq 1 .
$$

The solution of the second Diophantine equation is $v=3 a+2, a \geq 0$. This, when combined with the first equation, gives

$$
8 x=3 a+2,
$$

whose solution is $x=3 s+1, s \geq 0$. Hence, finally

$$
v=8 x=8(3 s+1) .
$$

Observe that, in Lemma 2.2, $p=5 v+1=3(8 s+3)$ is a multiple of 3, a result consistent with Corollary 2.1.

Using Lemma 2.2, we get the functions below :

$$
S S(42)=38, \operatorname{SS}(282)=278, \operatorname{SS}(402)=398, \operatorname{SS}(522)=518, \operatorname{SS}(642)=638 .
$$

Lemma 2.3 : Let $p$ be a prime of the form $p=5 v+1$ for some integer $v \geq 1$. Then,

$$
S S(p+1)=p-4,
$$

if $v=2(6 s+1)$ for some integer $s \geq 0$.

Proof: With $p=5 v+1$, we have

$$
(p+1) p \frac{(p-1)(p-2)(p-3)}{5 !}=(p+1) p \frac{v(5 v-1)(5 v-2)}{2 \times 3 \times 4} .
$$

Now, we consider the case when 3 divides $5 v-1$ and 4 divides $5 v-2$. Then,

$$
5 v=3 x+1,5 v=4 y+2 \text { for some integers } x \geq 1, y \geq 1 .
$$

The solutions of these two Diophantine equations are

$$
v=3 a+2=4 b+2 ; a \geq 0, b \geq 0 \text { being any integers. }
$$

This shows that $a=4 s, s \geq 1$. Therefore,

$$
v=3 a+2=2(6 s+1),
$$

which is the desired condition.

Lemma 2.3 gives the following functions :

$$
S S(12)=7, \operatorname{SS}(72)=65, \operatorname{SS}(132)=125, S S(192)=185, S S(252)=247 .
$$

The first example shows that Lemma 2.3 is valid for $s=0$ as well.

From the proof of Lemma 2.1, it may be deduced that, if $p=5 v+1$, then $S S(p+1)=p-2$ if and 
only if $v=6 s(s \geq 1)$. However, in other cases, there might be more than one solution, as the two lemmas below illustrate.

Lemma 2.4: Let $p$ be a prime of the form $p=5 v+1$ with $v=4(3 s+2), s \geq 0$. Then,

$$
S S(p+1)= \begin{cases}p-3, & \text { if } \quad s \text { is even } \\ p-4, & \text { if } s \text { is odd }\end{cases}
$$

Proof: Consider the expression

$$
(p+1) p \frac{(p-1)(p-2)(p-3)}{5 !}=(p+1) p \frac{v(5 v-1)(5 v-2)}{2 \times 3 \times 4} .
$$

Now, let $s$ be even, say, $s=2 r$ for some integer $r \geq 1$. Then,

$$
v=4(6 r+2)=8(3 r+1), 5 v-1=3(40 r+13) .
$$

Then, clearly $(p-1)(p-2)=5 v(5 v-1)$ is divisible by 4 !, and hence, $S S(p+1)=p-3$.

Next, let $s$ be odd of the form $s=2 t+1$ for some integer $t \geq 1$. Then,

$$
v=2(12 t+7), 5 v-1=3(40 t+13), 5 v-2=4(30 t+17) \text {. }
$$

In this case, $(p-1)(p-2)$ is not divisible by 4 !, but $(p-1)(p-2)(p-3)$ is divisible by $4 !$.

From Lemma 2.4, corresponding to $s=0$, we get the prime $p=41$ with $S S(42)=38$; when $s=1$, we get the prime $p=101$ which gives $S S(102)=97$. Again, with $s=2$, we get the prime $p=161$ with $S S(162)=158$. The next prime in the sequence is $p=281$ (corresponding to $s=4$ ) with $S S(282)=278$.

Lemma 2.5 : Let $p$ be a prime of the form $p=5 v+1$ with $v=2(3 s+1), s \geq 0$. Then,

$$
S S(p+1)= \begin{cases}p-3, & \text { if } s=4 t+1, \quad t \geq 0 \\ p-4, & \text { otherwise }\end{cases}
$$

Proof: We start with

$$
(p+1) p \frac{(p-1)(p-2)(p-3)}{5 !}=(p+1) p \frac{v(5 v-1)(5 v-2)}{2 \times 3 \times 4} .
$$

With $v=2(3 s+1),(p-1)(p-2)=30(3 s+1)(10 s+3)$.

When $s=4 t+1$, then $3 s+1=4(3 t+1)$, so that 4 ! divides $(p-1)(p-2)$. Thus, in this case,

$$
S S(p+1)=p-3 .
$$

Otherwise, 4 ! does not divide $(p-1)(p-2)$, but $(p-1)(p-2)(p-3)=60(3 s+1)(10 s+3)(15 s+4)$ is divisible by 5 !, so that $S S(p+1)=p-4$.

From Lemma 2.5, corresponding to $s=0$, we get the prime $p=11$ with $S S(12)=7 ; s=1$ gives the prime $p=41$, which is of the form $4 t+1$, so that $S S(42)=38$. Corresponding to $s=3$, we get the prime $p=101$ with $S S(102)=97$. Continuing, we get successively the functions $S S(132)=125$, 
$S S(192)=187, S S(252)=247$ and $S S(282)=278$.

Lemma 2.6 : Let $p$ be a prime of the form $p=5 v+1$ with $v=2(9 s+10), s \geq 0$. Then,

$$
S S(p+1)= \begin{cases}p-3, & \text { if } s \neq 4 t+2, t \geq 0 \\ p-4, & \text { otherwise }\end{cases}
$$

Proof: Consider the expression below :

$$
(p+1) p \frac{(p-1)(p-2)(p-3)}{5 !}=(p+1) p \frac{v(5 v-1)(5 v-2)}{2 \times 3 \times 4} .
$$

If $s=4 t+2$, then

$$
9 s+10=4(9 t+7)
$$

Therefore,

$$
(p-1)(p-2)=90(9 s+10)(10 s+11)
$$

which is divisible by $4 !$. Thus, in this case,

$$
S S(p+1)=p-3 .
$$

Otherwise, $(p-1)(p-2)$ is not divisible by 4 !, but 5 ! divides

$$
(p-1)(p-2)(p-3)=180(9 s+10)(10 s+11)(45 s+49)
$$

so that $S S(p+1)=p-4$.

Some functions, obtained from Lemma 2.6, are listed below :

$$
\begin{aligned}
& S S(102)=97, S S(192)=187, S S(282)=278, S S(462)=457, S S(642)=638, \\
& S S(822)=817, S S(912)=907, S S(1092)=1087, S S(1182)=1177, S S(1362)=1358 .
\end{aligned}
$$

Lemma 2.7 : Let $p$ be a prime of the form $p=5 v+1$ for some integer $v \geq 1$. Then,

$$
p-2 \leq S S(p+1) \leq p-4 \text {. }
$$

Proof: We prove the lemma by showing that $S S(p+1) \neq p-5$. So, we consider the expression below:

$$
(p+1) p \frac{(p-1)(p-2)(p-3)(p-4)}{6 !}=(p+1) p\left[\frac{v(5 v-1)(5 v-2)(5 v-3)}{2 \times 3 \times 4 \times 6}\right] .
$$

Now, we find the condition under which the term inside the square bracket on the right is an integer. To do so, first note that, by Corollary 2.1 , ( since 3 divides $p+1$ ), 9 must divide $5 v-1$. Then, since $5 v-3$ must be odd, we see that it remains dormant, and consequently, in such a case, we must have $S S(p+1) \leq p-4$.

The next three lemmas deal with the case when $p$ is a prime of the form $p=5 v+2$.

Lemma 2.8 : Let $p$ be a prime of the form $p=5 v+2$ for some integer $v \geq 1$. Then, 


$$
S S(p+1)=p-3,
$$

if $v=3(8 s+1)$ for some integer $s \geq 0$.

Proof: With $p=5 v+2$, we get

$$
(p+1) p \frac{(p-1)(p-2)}{4 !}=5(p+1) p \frac{v(5 v+1)}{2 \times 3 \times 4} .
$$

Now, consider the case when 8 divides $5 v+1$ while 3 divides $v$, so that

$5 v=8 x-1, v=3 y$ for some integers $x \geq 1, y \geq 1$.

The solution of the first equation is

$$
v=8 a+3, a \geq 0 .
$$

We are then lead to the equation

$$
8 a=3 y-3,
$$

with the solution $a=3(s+1), s \geq 0$. Plugging in this expression in $v=8 a+3$, we get the desired condition.

The following functions are obtained from Lemma 2.8 :

$$
S S(18)=14, S S(138)=134, \operatorname{SS}(258)=254, S S(618)=614, \operatorname{SS}(858)=854 .
$$

Lemma 2.9: Let $p$ be a prime of the form $p=5 v+2$ for some integer $v \geq 1$. Then,

$$
S S(p+1)=p-4
$$

if $v=3(4 s+3), s \geq 0$.

Proof: We start with

$$
(p+1) p \frac{(p-1)(p-2)(p-3)}{5 !}=(p+1) p \frac{v(5 v+1)(5 v-1)}{2 \times 3 \times 4} .
$$

Now, we consider the case when 4 divides $5 v-1$ and 3 divides $v$. Then,

$$
5 v=4 x+1, v=3 y \text { for some integers } x \geq 1, y \geq 1 .
$$

The first equation has the solution

$$
v=4 a+1, a \geq 0 .
$$

We are then faced with the Diophantine equation

$$
v=3 y=4 a+1,
$$

whose solution is

$$
a=3 s+2, s \geq 0 .
$$


After simplification, we get the condition desired.

From Lemma 2.9, we get the functions below.

$$
S S(48)=43, S S(108)=103, \operatorname{SS}(158)=153, S S(228)=223, S S(348)=343 .
$$

Lemma 2.10: Let $p$ be a prime of the form $p=5 v+2$ with $v=3(2 s+1), s \geq 0$. Then,

$$
S S(p+1)= \begin{cases}p-3, & \text { if } s \neq 4 t, \quad t \geq 0 \\ p-4, & \text { otherwise }\end{cases}
$$

Proof : Consider the expression below :

$$
(p+1) p \frac{(p-1)(p-2)(p-3)}{5 !}=(p+1) p \frac{v(5 v+1)(5 v-1)}{2 \times 3 \times 4} .
$$

Now, we consider the case when 2 divides $5 v+1$ and 3 divides $v$. Then,

$$
5 v=2 x-1, v=3 y \text { for some integers } x \geq 1, y \geq 1 .
$$

The first Diophantine equation has the solution

$$
v=2 a+1, a \geq 0,
$$

which, combined with the second equation, leads to

$$
3 y=2 a+1,
$$

which gives

$$
y=2 s+1, s \geq 0 .
$$

And finally, we get $v=3(2 s+1)$.

Now, since

$$
5 v+1=2(15 s+8)
$$

it follows that

$$
S S(p+1)=p-4, \text { if } 4 \text { divides } 15 s+8
$$

otherwise, $S S(p+1)=p-5$.

Now, noting that 4 divides $15 s+8$ if and only if $s=4 t, t \geq 1$, the lemma is established.

Some functions, obtained from Lemma 2.10, are

$$
\begin{aligned}
& S S(18)=14, S S(48)=43, S S(108)=103, S S(138)=134, S S(168)=163, \\
& S S(198)=193, S S(228)=223, S S(258)=254 .
\end{aligned}
$$

Lemma 2.11 - Lemma 2.14 consider the case when the prime $p$ is of the form $p=5 v+3$. Lemma 2.11 : Let $p$ be a prime of the form $p=5 v+3$, where $v=2(12 s+11), s \geq 0$. Then, 


$$
S S(p+1)=p-3 .
$$

Proof: Letting $p=5 v+3$ in

$$
(p+1) p \frac{(p-1)(p-2)}{4 !},
$$

we get

$$
(p+1) p\left[\frac{(5 v+2)(5 v+1)}{2 \times 3 \times 4}\right] \text {. }
$$

Now, in order that the above number is an integer, 8 must divide $5 v+2$, and 3 must divide $5 v+$ 1. This leads to the following two Diophantine equations

$$
5 v=8 x-2,5 v=3 y-1 \text { for some integers } x(\geq 1) \text { and } y(\geq 1),
$$

with solutions

$$
v=8 a+6, v=3 b+1(a \geq 1 \text { and } b \geq 1 \text { being integers }),
$$

respectively. Now, combining together, the resulting equation is

$$
8 a=3 b-5,
$$

whose solution is

$$
a=3 s+2, s \geq 0 .
$$

Hence,

$$
v=8(3 s+2)+6=2(12 s+11),
$$

which is the desired expression we were looking for.

Applying Lemma 2.11, we get the expressions below :

$$
S S(114)=110, S S(234)=230, S S(594)=590 .
$$

Lemma 2.12: Let $p$ be a prime of the form $p=5 v+3, v=4(3 s+1)$ for some integer $s \geq 0$. Then,

$$
S S(p+1)=p-4 \text {. }
$$

Proof: We start with

$$
(p+1) p \frac{(p-1)(p-2)(p-3)}{5 !}=(p+1) p\left[\frac{(5 v+2)(5 v+1) v}{2 \times 3 \times 4}\right] .
$$

We consider the case when 3 divides $5 v+1,2$ divides $5 v+2$ and $v$ itself is a multiple of 4 . Then, we have

$$
5 v=3 x-1,5 v=2 y-2, v=4 z \text { for some integers } x(\geq 1), y(\geq 1) \text { and } z(\geq 1) .
$$

The first Diophantine equation gives the solution 


$$
v=3 a+1, a \geq 1 .
$$

This, together with the condition $v=4 z$, requires that

$$
3 a=4 z-1,
$$

whose solution is

$$
a=4 s+1, s \geq 1 .
$$

Therefore,

$$
v=3 a+1=3(4 s+1)+1=4(3 s+1) .
$$

Applying Lemma 2.12, we get the functions below :

$S S(24)=19, S S(84)=79, S S(264)=259, S S(384)=379$.

Lemma 2.13: Let $p$ be a prime of the form $p=5 v+3, v=8(3 s+2)$ for some integer $s \geq 1$. Then,

$$
S S(p+1)=p-4 .
$$

Proof: We start with the following simplified form:

$$
(p+1) p \frac{(p-1)(p-2)(p-3)}{5 !}=(p+1) p\left[\frac{(5 u+2)(5 u+1) u}{2 \times 3 \times 4}\right] .
$$

Now, we consider the case when 3 divides $5 v+1$ and 8 divides $v$. Then,

$$
5 v=3 x-1, v=8 y \text { for some integers } x(\geq 1) \text { and } y(\geq 1) .
$$

The first equation has the solution $v=3 a+1, a(\geq 1)$, and hence, we have to consider

$$
3 a=8 y-1,
$$

whose solution is $a=8 s+5, s \geq 1$.

Therefore,

$$
v=3(8 s+5)+1=8(3 s+2),
$$

which we intended to establish.

Application of Lemma 2.13 gives the functions :

$$
S S(84)=79, \operatorname{SS}(444)=439 .
$$

Lemma 2.14: Let $p$ be a prime of the form $p=5 v+3$, where $v=2(3 s+2)$ for some integer $s \geq 1$ such that $s \neq 4 t+3$ for any $t \geq 0$. Then,

$$
S S(p+1)=p-4 .
$$

Proof: We start with 


$$
(p+1) p \frac{(p-1)(p-2)(p-3)}{5 !}=(p+1) p\left[\frac{(5 v+2)(5 v+1) v}{2 \times 3 \times 4}\right] .
$$

We consider the case when 3 divides $5 v+1$ and 2 divides $v$. Then,

$$
5 v=3 x-1, v=2 y \text { for some integers } x(\geq 1) \text { and } y(\geq 1) .
$$

The solution of the first equation is

$$
v=3 a+1, a \geq 1 .
$$

Then, the combined Diophantine equation is

$$
2 y=3 a+1,
$$

with the solution

$$
y=3 s+2, s \geq 0 .
$$

Therefore,

$$
v=3(8 s+5)+1=8(3 s+2) .
$$

Note that, $5 v+2=2(15 s+11)$, and hence, to complete the proof, we have to guarantee that 4 does not divide $15 s+11$. To do so, we consider the equation $15 s=4 b-11$, which has the solution

$$
s=4 t+3, t \geq 0 .
$$

Thus, if $s \neq 4 t+3$ for any $t \geq 0$, then $\operatorname{SS}(p+1)=p-4$.

Hence, the proof of the lemma is complete.

Lemma 2.14 gives the following functions

$$
S S(24)=19, \operatorname{SS}(54)=49, \operatorname{SS}(84)=79, \operatorname{SS}(174)=169, S S(264)=259 .
$$

It may be mentioned here that, if $s=4 t+3(t \geq 0)$ in Lemma 2.14, then by Lemma 2.11,

$$
S S(p+1)=p-3 .
$$

Finally, we have the following lemma, dealing with the case when $p$ is a prime of the form

$$
p=5 v+4
$$

Lemma 2.15: Let $p$ be a prime of the form $p=5 v+4, v=24 s+17$ for some $s \geq 0$. Then,

$$
S S(p+1)=p-3 .
$$

Proof: With $p=5 v+4$, we get

$$
(p+1) p \frac{(p-1)(p-2)}{4 !}=(p+1) p\left[\frac{(5 v+3)(5 v+2)}{2 \times 3 \times 4}\right] .
$$

We consider the case when 8 divides $5 v+3$ and 3 divides $5 v+2$. Thus, 


$$
5 v+3=8 x, 5 v+2=3 y \text { for some integers } x(\geq 1) \text { and } y(\geq 1),
$$

that is, $5 v=8 x-3,5 v=3 y-2$.

The solutions of the above two equations are

$$
v=8 a+1, v=3 b+2 ; a \geq 1 \text { and } b \geq 1 \text { being integers. }
$$

Now, combining together the above two Diophantine equations, we get the equation

$$
8 a=3 b+1,
$$

with the solution

$$
a=3 s+2, s \geq 0 .
$$

Thus,

$$
v=8(3 s+2)+1=24 s+17,
$$

which is the condition desired.

Some of the functions found from Lemma 2.15 are given below.

$$
S S(90)=86, S S(450)=446, S S(570)=566, S S(810)=806 .
$$

Remark 2.1 : In the proof of Lemma 2.15, writing the two equations in (1) in the form

$$
5 v+3=8 x=3 y+1,
$$

we get the solution

$$
x=3 t+2, t \geq 0 .
$$

Then,

$$
p=5 v+4=8 x+1=8(3 t+2)+1=24 t+17 .
$$

Having the solution in the above form, we get the functions below :

$$
\begin{aligned}
& S S(18)=14, S S(42)=38, S S(90)=86, S S(114)=110, S S(138)=134, \\
& S S(234)=230, S S(258)=254, S S(282)=278, S S(354)=350 .
\end{aligned}
$$

\section{REFERENCES}

[1] Sandor, J. (2001). On a New Smarandache Type Function, Smarandache Notions Journal, 12: 247248.

[2] Majumdar, A.A.K. (2018). Smarandache Numbers Revisited, Pons Publishing House, Belgium, (available at http://fs.gallup.unm.edu/SmarandacheNumbers.pdf). 